\title{
Vereinfachte Berechnung der Radioaktivität und der zugehörigen Streuung (inkl. Vertrauensintervall und Vertrauensgrenzen) mit Hilfe eines speziellen Rechenschiebers und passender Rechentabellen
}

Von M. M. Bezzegh-Galántai und H. L. Le Roy 


\title{
Vereinfachte Berechnung der Radioaktivität und der zugehörigen Streuung (inkl. Vertrauensintervall und Vertrauensgrenzen) mit Hilfe eines speziellen Rechenschiebers und passender Rechentabellen
}

\author{
Von M. M. Bezzegh-Galántai ${ }^{1}$ ) und H. L. Le RoY ${ }^{2}$ ) \\ Manuskript eingegangen am 14. Juli 1965
}

\section{Einleitung}

In einer früheren Arbeit (BEZzEGH-GaLÁnTAI und LE Roy) ${ }^{3}$ ) wurde eine vereinfachte Methode für die Berechnung der Radioaktivität und ihrer Streuung (mit Hilfe von graphischen Darstellungen) beschrieben. In der Folge soll nun gezeigt werden, wie ein speziell für diese Berechnung entwickelter Rechenschieber in Verbindung mit passenden Rechentabellen die eingehende Datenanalyse ohne grossen Zeitaufwand realisieren lässt. Dieses Vorgehen bietet gegenüber dem graphischen Verfahren den Vorteil, dass es zu allen Apparaten direkt verwendbar ist. Die Messungen können sowohl mit vorgewählter Impulszahl als auch mit vorgewählter Zeit (einmalige oder mehrmalige Messung) durchgeführt werden.

\section{Rechenschieber}

Auf dem Rechenschieber sind die Funktionen auf speziellen Skalen aufgetragen, welche für die Berechnung der Impulsfrequenzen, Aktivitäten und Streuungen unter Berücksichtigung des Untergrundes benötigt werden. Diese besonderen Funktionen sind in den Rechentabellen in jenen Feldern zu finden, die das Symbol $\bullet$ aufweisen. Abb. 1 illustriert die Struktur des Rechenschiebers. Die Skalen $\sqrt{x+1}, \sqrt{x^{2}+1}$, $\sqrt{(x+1) /(x-1)^{2}}$ usw. sind so zu verstehen, dass die Grösse $x$ nur als Leitsymbol für numerische Werte, zum Beispiel für $n, \bar{n}, n_{0}, \bar{n}_{0}, t, \bar{t}, t_{0}, \bar{t}_{0}, a_{n}, a_{n}, a_{t}$ und $a_{\bar{t}}$ (siehe Tabellen 1-6), eingeführt wird. Die zur Diskussion stehenden, etwas komplexen Zürich

1) Eidg. Anstalt für Wasserversorgung, Abwasserreinigung und Gewässerschutz an der ETH,

2) Laboratorium für Biometrik und Populationsgenetik am Institut für Tierzucht der ETH, Zürich.

3) Vereinfachte Berechnung der Radioahtivität und ihrer Streuung im Wasser für ein festgelegtes Avbeitsschema, Schweiz. Z. Hydrol. 23, 494 (1961). 

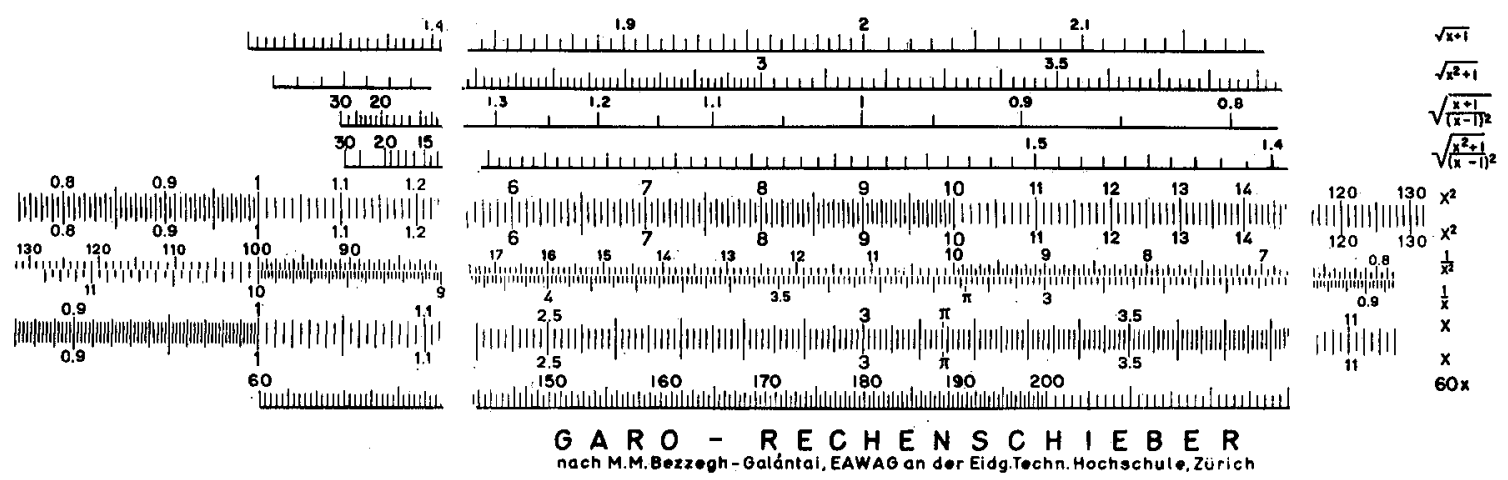

Abbildung 1

Funktionen können somit aus dem Rechenschieber unmittelbar abgelesen und in die Rechentabellen eingesetzt werden.

\section{Rechentabellen}

Die Rechentabellen sind durchwegs wie folgt aufgebaut:

a) Der obere, fest umrandete Teil dient der Aufnahme der Daten, die für die Auswertung unbedingt benötigt werden. Für wiederholte Messungen ist in den Tabellen 4, 5 und 6 am Tabellenkopf noch eine Tabelle vorgesehen, die direkt die einzelnen Messwerte aufnehmen kann und zur notwendigen Mittelwertsberechnung gute Dienste leisten dürfte.

b) Der linksseitige, festumrandete Tabellenteil enthält $Z$ wischenergebnisse, die mit Hilfe des Rechenschiebers berechnet werden können. Hier finden sich alle speziellen Funktionen, die unter 2. erwähnt wurden.

c) Die im rechtsseitig anschliessenden Tabellenteil aufgeführten Ergebnisse folgen aus den linksseitigen Daten durch kolonnenweise Multiplikation, wie dies im Tabellenkopf unter Rechenvorschrift vermerkt ist.

d) Der untere Tabellenteil dient der numerischen Charakterisierung der Reproduktionsfähigkeit der Ergebnisse auf Grund des prozentualen Vertrauensintervalles bzw. der 5\%-Vertrauensgrenzen.

e) Schlussendlich ist eine Rubrik für die Kennzeichnung des analysierten Materials vorgesehen. Zudem sind Angaben über die an der Auswertung beteiligten Personen (inklusive Daten) möglich.

\section{Auswertung, Symbole}

a) Die Tabellen dienen zur Berechnung der folgenden Grössen (inklusive Vertrauensintervall und Vertrauensgrenzen):

Tabelle 1: Bruttoimpulsfrequenz $N^{B}$ und Bruttoaktivität $A^{B}$ bei einmaliger Messung $(r=1), n$ (Impulse) oder $t$ (Zeitspanne) vorgegeben. 
Tabelle 2: Nettoimpulsfrequenz $N^{\prime}$ und Nettoaktivität $A^{\prime}$ bei einmaliger Messung $(r=1), n$ (Impulse) vorgegeben.

Tabelle 3: Nettoimpulsfrequenz $N^{\prime}$ und Nettoaktivität $A^{\prime}$ bei einmaliger Messung $(r=1), t$ (Zeitspanne) vorgegeben.

Tabelle 4: Wie Tabelle 1, jedoch mit mehrmaliger Messung $(r>1)$.

Tabelle 5: Wie Tabelle 2, jedoch mit mehrmaliger Messung $(r>1)$.

Tabelle 6: Wie Tabelle 3, jedoch mit mehrmaliger Messung $(r>1)$.

b) Weitere, in den Tabellen benützte Symbole:

$C=A_{E} / \bar{N}_{E}=$ Konstante, wobei $A_{E}$ die Aktivität des verwendeten Eichpräpaparates und

$\bar{N}_{E}$ den Durchschnittswert der am Eichpräparat

$t_{p}=$ Zeitspanne für das Präparat, gemessenen Impulsfrequenzen bedeuten.

$t_{o}=$ Zeitspanne für den Untergrund,

$n_{p}=$ Impulse für das Präparat,

$n_{o}=$ Impulse für den Untergrund,

$\sigma_{j}=$ Standardabweichung, wobei $j=N^{B}$, bzw. $A^{B}$ oder

$N^{\prime}$, bzw. $A^{\prime}$ oder

$\bar{N}^{B}$, bzw. $\overline{A^{B}}$ oder

$\delta=\sigma_{j} / j=$ relative Standardabweichung,

$\bar{N}^{\prime}$, bzw. $\bar{A}^{\prime}$

$P=$ Irrtumwahrscheinlichkeit (Fehler erster Art).

Ferner ist zu beachten:

a) Will man nur Auskunft über die Impulsfrequenz bzw. Aktivität haben, so genügt es, wenn die Berechnung auf die Zeilen $Z_{1}$ und $Z_{2}$ beschränkt wird.

b) Sind die Vertrauensgrenzen zu den absoluten Werten erwünscht, so ist die Berechnung auf die Zeilen $Z_{1}, Z_{2}, Z_{3}$ auszudehnen. Anschliessend sind im unteren Tabellenteil die Felder (rechts) für die Vertrauensgrenzen (VG) zu berücksichtigen.

c) Interessiert nur das prozentuale Vertrauensintervall sowie die Impulsfrequenz bzw. die Aktivität, so beschränkt sich die Berechnung auf die Zeilen $Z_{1}, Z_{2}, Z_{4}$ und $Z_{\mathbf{5}}$. Anschliessend ist im unteren Tabellenteil (links) das Feld für die numerische Kennzeichnung der prozentualen Vertrauensintervalle auszufüllen.

d) Die in den Tabellen 1-6 aufgeführten Rechenvorschriften für Aktivitäten $\left(A^{B}\right.$. bzw. $A^{\prime}$ ) sind gültig, wenn das Bezugspräparat dieselbe Energie und dieselbe Schichtdicke aufweist wie die zu messende Probe. In diesem Falle (relative Messung!) ist der Selbstabsorptionsfaktor $=1$. Weicht jedoch die Energie oder die Schichtdicke des zu messenden Präparates von derjenigen des Bezugspräparates ab, so muss der jeweilige Selbstabsorptionsfaktor mitberücksichtigt werden.

e) Die Skalen $\sqrt{x+1}, \sqrt{x^{2}+1}$ usw. auf dem Rechenschieber gelten nur für den Bereich $1,1<x<10$. $x$-Werte kleiner als 1,1 in $\sqrt{x+1}, \sqrt{x^{2}+1}$ usw. haben keine Bedeutung, da bei diesen Fällen die Aktivitäten ein so geringes Ausmass erreichen, dass sie mit den Messgeräten nicht erfasst werden können. $x$-Werte grösser als 10 sind möglich; durch geschickte Wahl der Messanordnung gelingt es jedoch leicht, einen $x$-Wert im Intervall $1,1<x<10$ zu realisieren. Die Tabellen 1 bis 6 dagegen haben Gültigkeit für beliebige $x$-Werte. 
Tab.1: $N^{B}$ bzw. $A^{B}$, $n$ oder t fix und $r=1$

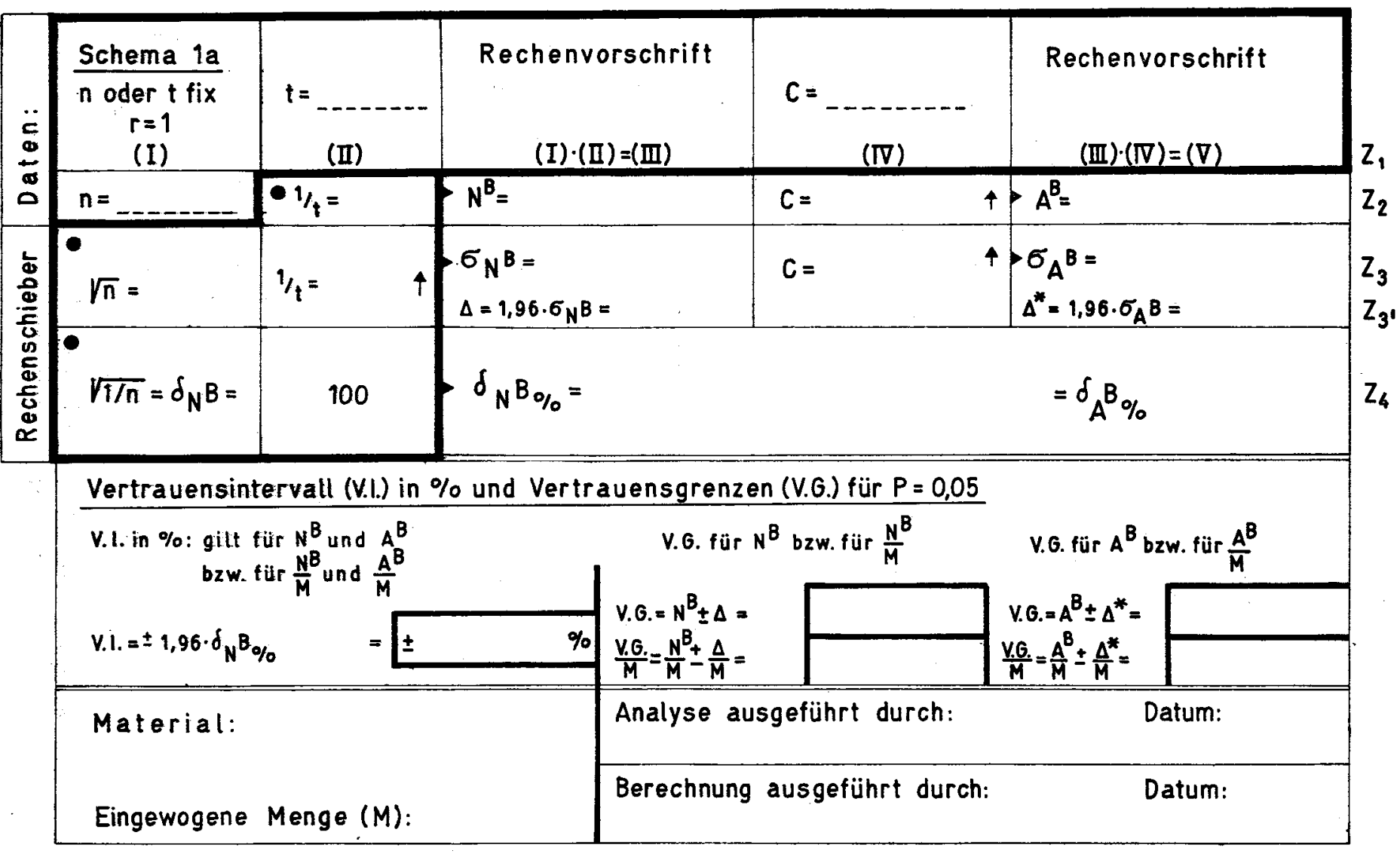


Tab. 2: $N^{\prime}$ bzw. $A^{\prime}, n$ fix und $r=1$

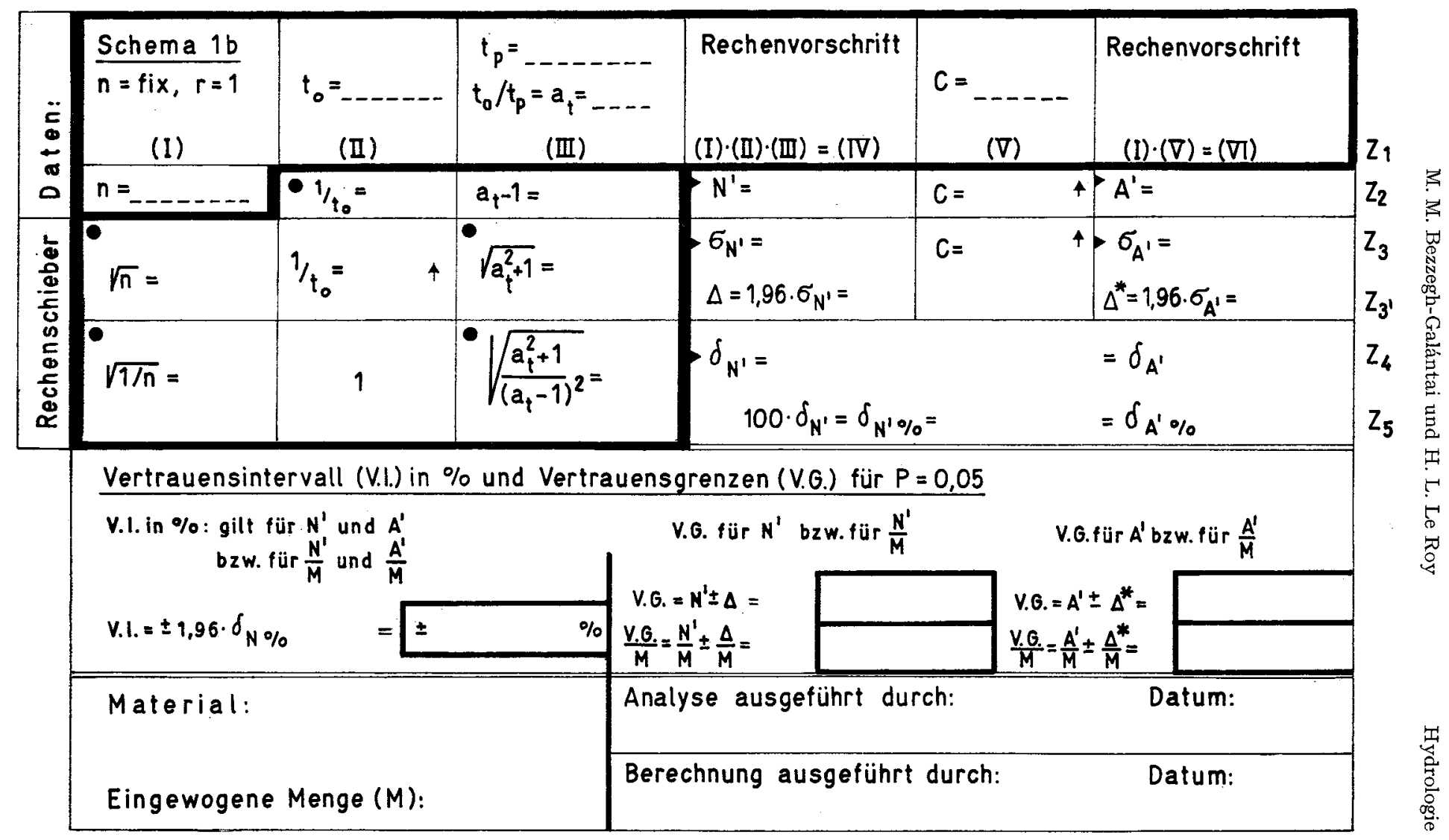


Tab. 3: $N^{\prime}$ bzw. $A^{\prime}$, ffix und $r=1$

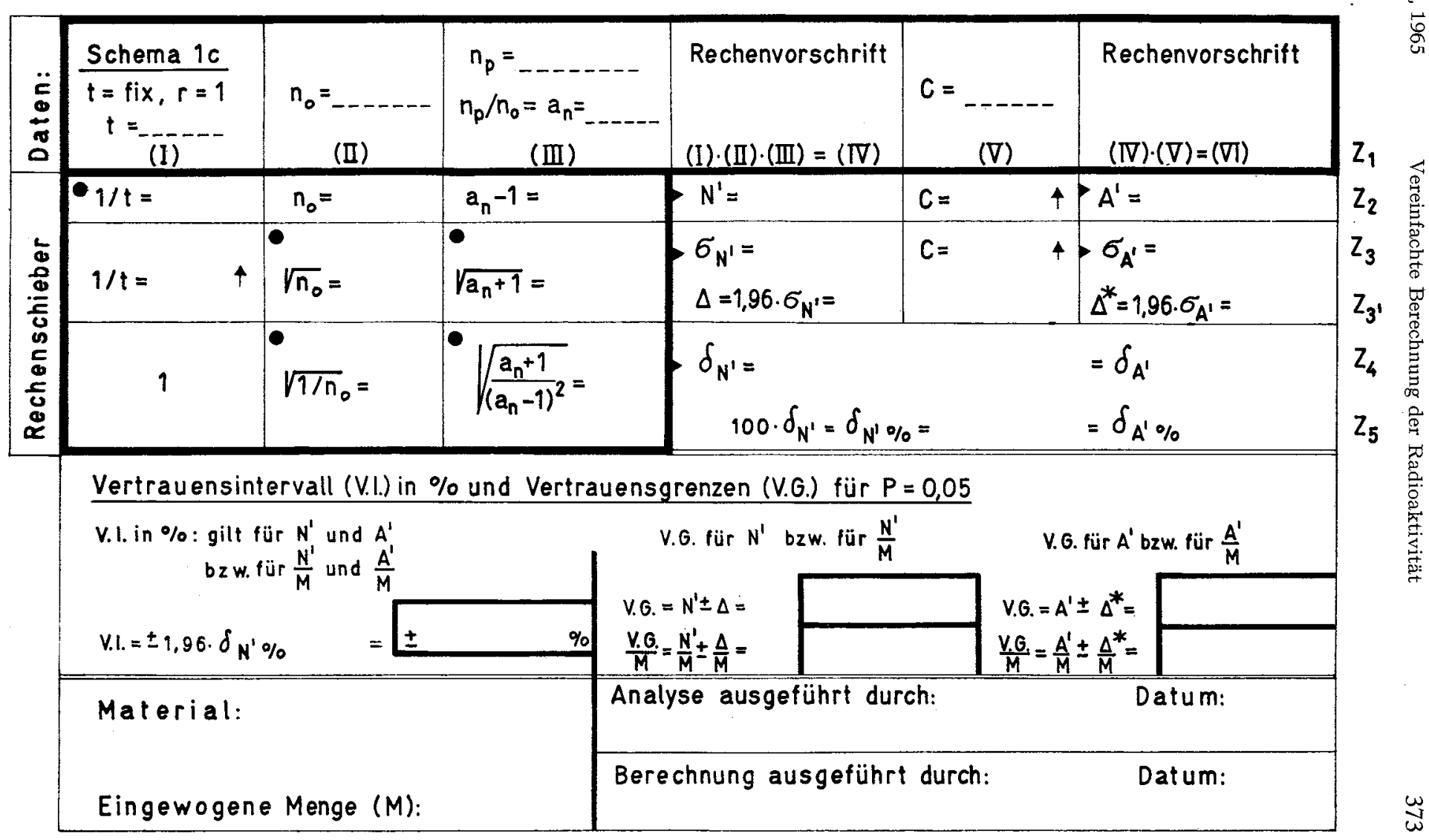


Tab. 4: $\bar{N}^{B}$ bzw. $\bar{A}^{B}, n$ oder $t$ fix und $r>1$ Urliste (Einzetmessungen)

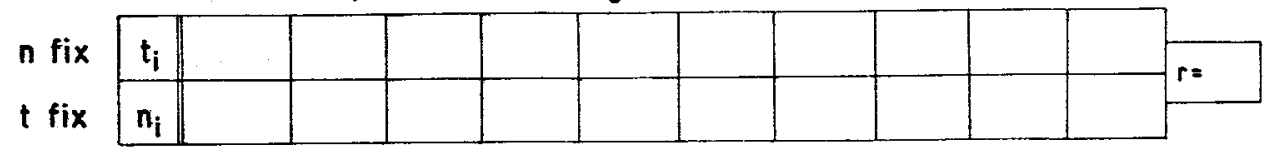

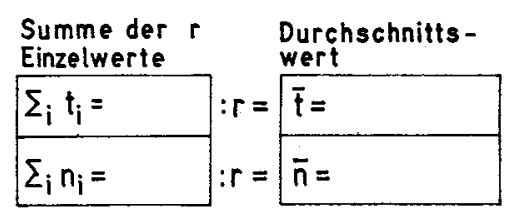

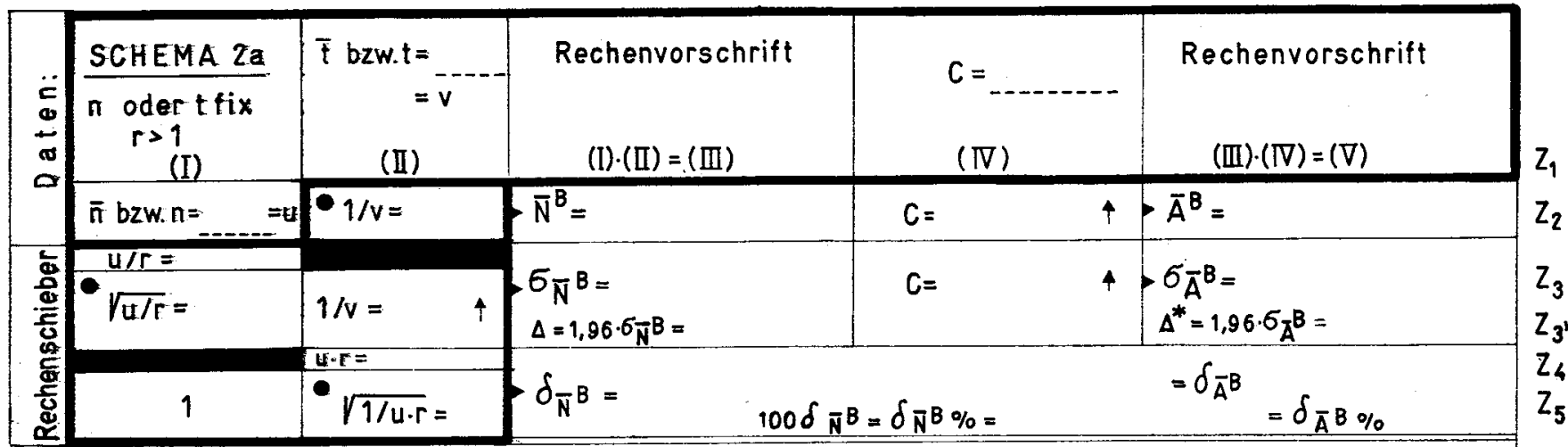

Vertrauensintervall (V.I.) in \% und Vertrauensgrenzen(V.G.) für $P=0,05$

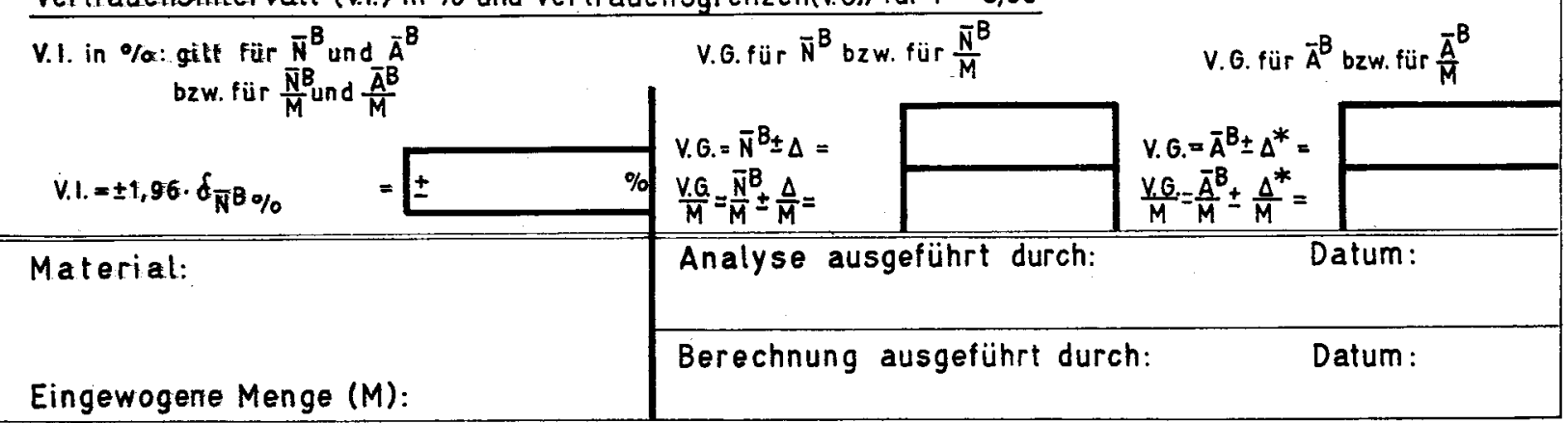


Tab. $5: \bar{N}^{\prime}$ bzw. $\vec{A}^{\prime}, n$ fix und $r,>1$

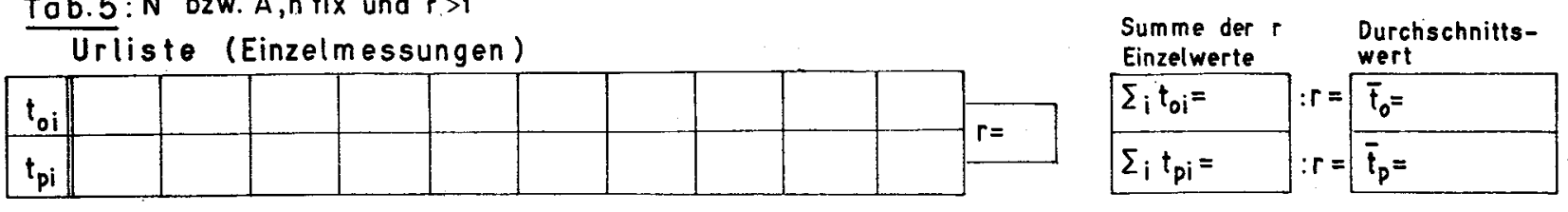

\begin{tabular}{|c|c|c|c|c|c|c|}
\hline$\underset{\sigma}{\stackrel{C}{c}}$ & $\begin{array}{c}\text { SCHEMA 2b } \\
r>1, \text { fix } \\
\text { (I) }\end{array}$ & $\begin{array}{c}\bar{t}_{0}=\ldots \\
\text { (II) }\end{array}$ & $\begin{array}{l}\bar{t}_{p}=\ldots \\
\bar{t}_{0} / \bar{t}_{p}=a_{\bar{t}}= \\
(\mathbb{W})^{-\ldots}\end{array}$ & $\begin{array}{l}\text { Rechenvorschrift } \\
\text { (I) } \cdot \text { (II) } \text { (III) }=\text { (IV) }\end{array}$ & $\begin{array}{l}C= \\
(\nabla)\end{array}$ & 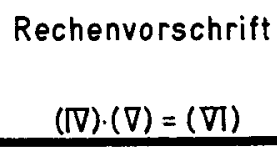 \\
\hline 0 & $n=\ldots$ & $1 / \bar{t}_{0}=$ & $a_{\bar{i}}-1=$ & $\bar{N}^{\prime}=$ & $C=\quad \uparrow$ & $\bar{A}^{\prime}=$ \\
\hline 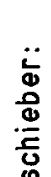 & $\frac{n / r=}{\sqrt{n / r}=}$ & $1 / \bar{t}_{0}=4$ & $\sqrt{a_{\frac{1}{t}}^{2}+1}=$ & $\begin{array}{l}\sigma_{\bar{N}^{\prime}}= \\
\Delta=1,96 \cdot \sigma_{N^{\prime}}=\end{array}$ & $\uparrow$ & $\begin{array}{l}\sigma_{\bar{A}^{\prime}}= \\
\Delta^{*}=1,96 \cdot \sigma_{\bar{A}^{i}}=\end{array}$ \\
\hline 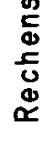 & 1 & $\sqrt{\frac{1}{n \cdot r}}=$ & $\sqrt{\frac{a_{\bar{f}}^{2}+1}{\left(a_{\bar{t}}-1\right)^{2}}}=$ & $\begin{array}{l}\delta_{\bar{N}^{\prime}}= \\
100 \cdot \delta_{\bar{N}^{\prime}}=\delta\end{array}$ & & $\begin{array}{l}=\delta_{\bar{A}^{\prime}} \\
=\delta_{\bar{A}^{\prime} \%}\end{array}$ \\
\hline
\end{tabular}

Vertrauensintervall (V.I.) in \% und Vertrauensgrenzen (V.G.) für $P=0,05$

V.l. in $\%$ : gilt für $\bar{N}^{\prime}$ und $\bar{A}^{\prime}$ bzw. für $\frac{N^{\prime}}{M}$ und $\frac{\bar{A}}{M}$ V.I. $= \pm 1,96 \cdot \delta \bar{N}^{\prime} \%$

$= \pm$
V. G. für $\bar{N}^{\prime}$ bzw. $\frac{\bar{N}^{\prime}}{M}$ V.G. für $A^{\prime}$ bzw. für $\frac{\bar{A}^{\prime}}{M}$

\begin{tabular}{|l|l|l|}
\hline Material: & $\frac{V G G}{M}=\frac{\bar{N}^{\prime}}{M} \pm \frac{\Delta}{M}=$ & $\frac{V, G}{M}=\frac{\bar{A}^{\prime}}{M} \pm \frac{\Delta^{*}}{M}=$ \\
\hline \multirow{2}{*}{ Eingewogene Menge $(M):$} & Analyse ausgeführt durch: & Datum: \\
\cline { 2 - 3 } & Berechnung ausgeführt durch: & Datum: \\
\hline
\end{tabular}


Tab. 6: $\bar{N}^{\prime}$ bzw. $\bar{A}^{\prime},+$ fix und $r>1$

Summe der $r$

Durchsehnitts-
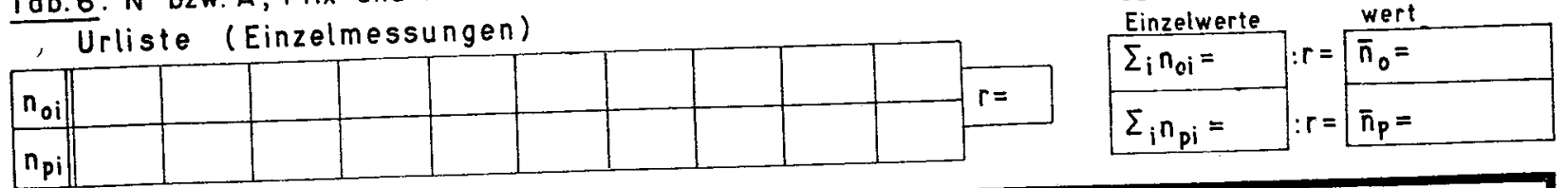

\begin{tabular}{|c|c|c|c|c|c|c|c|}
\hline$\frac{c}{\Phi}$ & $\begin{array}{c}\text { SCHEMA } 2 C \\
r>1, t f f x \\
t= \\
\text { (I) }\end{array}$ & $\begin{array}{r}\overline{\mathrm{n}}_{0}=\ldots \\
\text { (II) }\end{array}$ & $\begin{array}{c}\bar{n}_{p}= \\
\bar{n}_{p} / \bar{n}_{0}=a_{\bar{n}}= \\
\text { (III) }\end{array}$ & $\begin{array}{l}\text { Rechenvorschrift } \\
\text { (I) } \cdot \text { (II) } \cdot \text { (III) }=(\text { IV })\end{array}$ & $\begin{array}{l}C= \\
(\nabla)\end{array}$ & & $\begin{array}{l}\text { Rechenvorschrift } \\
(I V) \cdot(\nabla)=(\nabla))\end{array}$ \\
\hline & - $1 / t=$ & $\bar{n}_{0}=$ & $a_{\bar{n}}-1=$ & $\bar{N}^{\prime}=$ & $C=$ & $\uparrow$ & $\bar{A}^{\prime}=$ \\
\hline 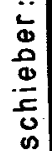 & $1 / t=\uparrow$ & $\sqrt{\pi_{0} / r}=$ & $\sqrt{a_{n}+1}=$ & $\begin{array}{l}\sigma_{\bar{N}^{\prime}}= \\
\Delta=1,96 \cdot \sigma_{\bar{N}^{\prime}}=\end{array}$ & $C=$ & $\uparrow$ & $\left\{\begin{array}{l}\sigma_{\bar{A}^{i}}= \\
\Delta^{*}=1,96 \cdot \sigma_{\bar{A}^{i}}=\end{array}\right.$ \\
\hline 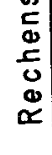 & 1 & $\begin{array}{l}\bar{n}_{0} \cdot r= \\
\sqrt{1 / \bar{n}_{0} \cdot r}=\end{array}$ & $\sqrt{\frac{a_{n}+1}{\left(a_{n}-1\right)^{2}}}=$ & $\begin{array}{l}\delta_{\bar{N}^{\prime}}= \\
100 \cdot \delta_{\bar{N}^{\prime}}=\end{array}$ & & & $\begin{array}{l}=\delta \bar{A}^{\prime} \\
=\delta_{\bar{A}^{\prime} \%}\end{array}$ \\
\hline
\end{tabular}

$z_{1}$

Vertrauensintervall (V.I.) in \% und Vertrauensgrenzen (V.G.) für $P=0,05$

V.t. in \% : gilt für $\bar{N}^{\prime}$ und $\bar{A}^{\prime}$

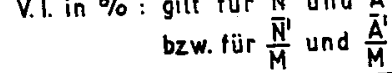

V. $I .= \pm 1,96 \cdot \delta_{\bar{N}^{\prime} \%}$

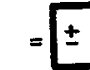

V.G. für $\bar{N}^{\prime}$ bzw: $\frac{N}{M}$

V. G.für $\bar{A}^{\prime}$ bzw. für $\frac{\bar{A}^{\prime}}{M}$

Material:

V.G. $=N^{\prime} \pm \Delta=$

$\frac{V \cdot G .}{M}=\frac{N^{\prime}}{M} \pm \frac{\Delta}{M}=$

Analyse ausgeführt durch:

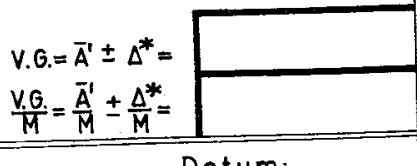

Berechnung ausgeführt durch:

Datum: 